\title{
Hernia de Bochdalek complicada con perforación gástrica en un paciente adulto. Reporte de un caso
}

\author{
Bochdalek hernia complicated with gastric perforation in an adult patient. \\ A case report
}

Lilia Melissa Cruz Cantú, ${ }^{*}$ Jorge Favio Lezama de Luna, ${ }^{* *}$ Carlos Manuel Sámano Robles**

\begin{abstract}
Palabras clave: Hernia de Bochdalek, hernia congénita, hernia diafragmática.

Key words: Bochdalek hernia, congenital hernia, diaphragmatic hernia.
\end{abstract}

* Médico residente del Servicio de Cirugía General.

** Médico adscrito al Servicio de Cirugía General.

Departamento de Cirugía General y Cirugía de Mínima Invasión, Hospital General de Zona con Medicina Familiar Núm. 6. Instituto Mexicano del Seguro Social.

Recibido: 11/03/2016 Aceptado: 25/07/2016

\section{RESUMEN}

La hernia de Bochdalek representa un defecto congénito en el diafragma que frecuentemente se diagnostica en el periodo neonatal. El tratamiento quirúrgico debe llevarse a cabo aun si el paciente se encuentra asintomático por el riesgo de estrangulación que existe. Informamos el caso de un individuo de sexo femenino de 43 años de edad con diagnóstico de hernia de Bochdalek complicada con perforación gástrica, así como su resolución satisfactoria por vía quirúrgica mediante un abordaje abdominal subcostal, cierre primario de la perforación gástrica y colocación de sonda intrapleural izquierda.

\section{ABSTRACT}

Bochdalek hernia is a congenital defect in the diaphragm frequently diagnosed in the neonatal period. Surgical treatment should be carried out even if the patient is asymptomatic because the risk of strangulation exists. We report the case of a 43-year-old female patient with a diagnosis of Bochdalek hernia complicated with gastric perforation, as well as its satisfactory surgical resolution through a subcostal abdominal approach, primary closure of the gastric perforation and catheterization with left thoracic tube.

\section{INTRODUCCIÓN}

$\mathrm{L}$ a hernia de Bochdalek fue descrita en 1847 por Alexander Bochdalek; se origina debido al cierre incompleto del canal pleuroperitoneal, así como por la falta de migración de la musculatura durante el periodo embrionario, lo que resulta en un defecto diafragmático posterolateral. ${ }^{1}$ Es una anomalía congénita poco común que se presenta en uno de cada 2,000 a 5,000 nacidos vivos. ${ }^{2}$ Sin embargo, su frecuencia en la vida adulta aún no está bien establecida; existen series que la informan hasta en $1.3 \%$, con predominio del lado izquierdo en 80 a $90 \% .^{3-5}$ Menos de 20 casos con complicaciones relacionadas con perforación gástrica han sido publicados en la literatura. ${ }^{6} \mathrm{Su}$ tratamiento debe de ser quirúrgico en cualquier momento del diagnóstico. 2,3,7

Informamos el caso de una persona de sexo femenino de 43 años de edad con diagnóstico de hernia de Bochdalek con presencia de perforación en el estómago migrado hacia el tórax, así como su resolución por vía quirúrgica mediante un abordaje abdominal subcostal y colocación de sonda intrapleural izquierda.

\section{REPORTE DEL CASO}

Femenina de 43 años de edad sin antecedentes familiares ni personales médicos de importancia y sin historia de trauma; fue admitida al Servicio de Urgencias de nuestro hospital por un cuadro de dolor abdominal intermitente en la fosa iliaca izquierda con irradiación a la región lumbar ipsilateral, asociado con náusea y vómitos de contenido gastrobiliar y sensación de plenitud postprandial, con accesos de tos ocasionales, de tres semanas de evolución, sin otra sintomatología agregada; signos vitales y exámenes de laboratorio de rutina dentro de parámetros normales. A la exploración física, presentó hipoventilación basal pulmonar izquierda y matidez a la percusión, sin otro agregado; el abdomen con peristalsis presente y adecuada, blando, depresible, doloroso a la 
palpación en el flanco y fosa iliaca izquierdos, sin datos de irritación peritoneal; resto, asignológico. Se realizó una tomografía axial computada toracoabdominal contrastada en cortes sagital y coronal, y se encontró un defecto de continuidad en la porción posterolateral del diafragma izquierdo, con herniación del estómago e intestino delgado y pulmón izquierdo hipoplásico (Figura 1).

La mujer fue programada para cirugía de urgencia por presentar inestabilidad hemodinámica asociada con dolor súbito a nivel del epigastrio con irradiación al tórax y zona lumbar izquierda que no cedía a la administración de analgésicos, con datos de irritación peritoneal a la exploración física abdominal, sin mejoría a la reanimación inicial. Se realizó una incisión subcostal bilateral, y se encontró un defecto de aproximadamente $10 \mathrm{~cm}$ en la porción posterolateral del diafragma izquierdo. Se procedió a reducir el estómago e intestino delgado del tórax hacia la cavidad abdominal; se halló una perforación gástrica de aproximadamente 5 cm al nivel de la curvatura mayor. Se realizó el cierre primario de la perforación en dos planos con sutura absorbible y puntos de Lembert con sutura de seda (Figura 2). En nuestro hospital no contamos con mallas protésicas por lo que se decidió realizar ante urgencia el cierre primario de defecto diafragmático con sutura no absorbible

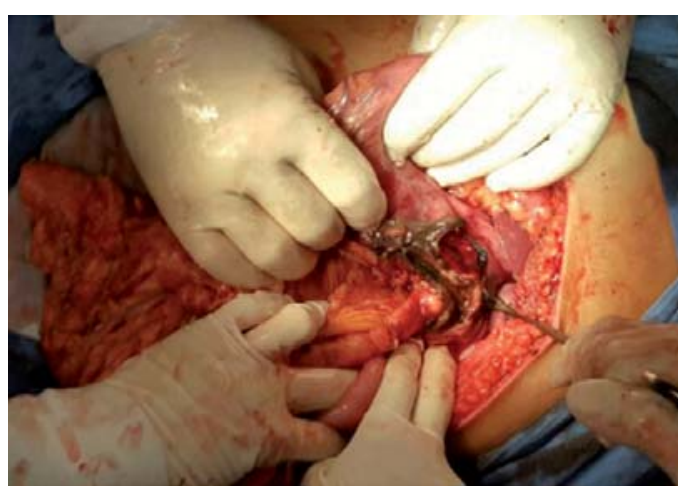

Figura 2. Abordaje subcostal abdominal. Se observa el defecto posterolateral en el diafragma izquierdo.
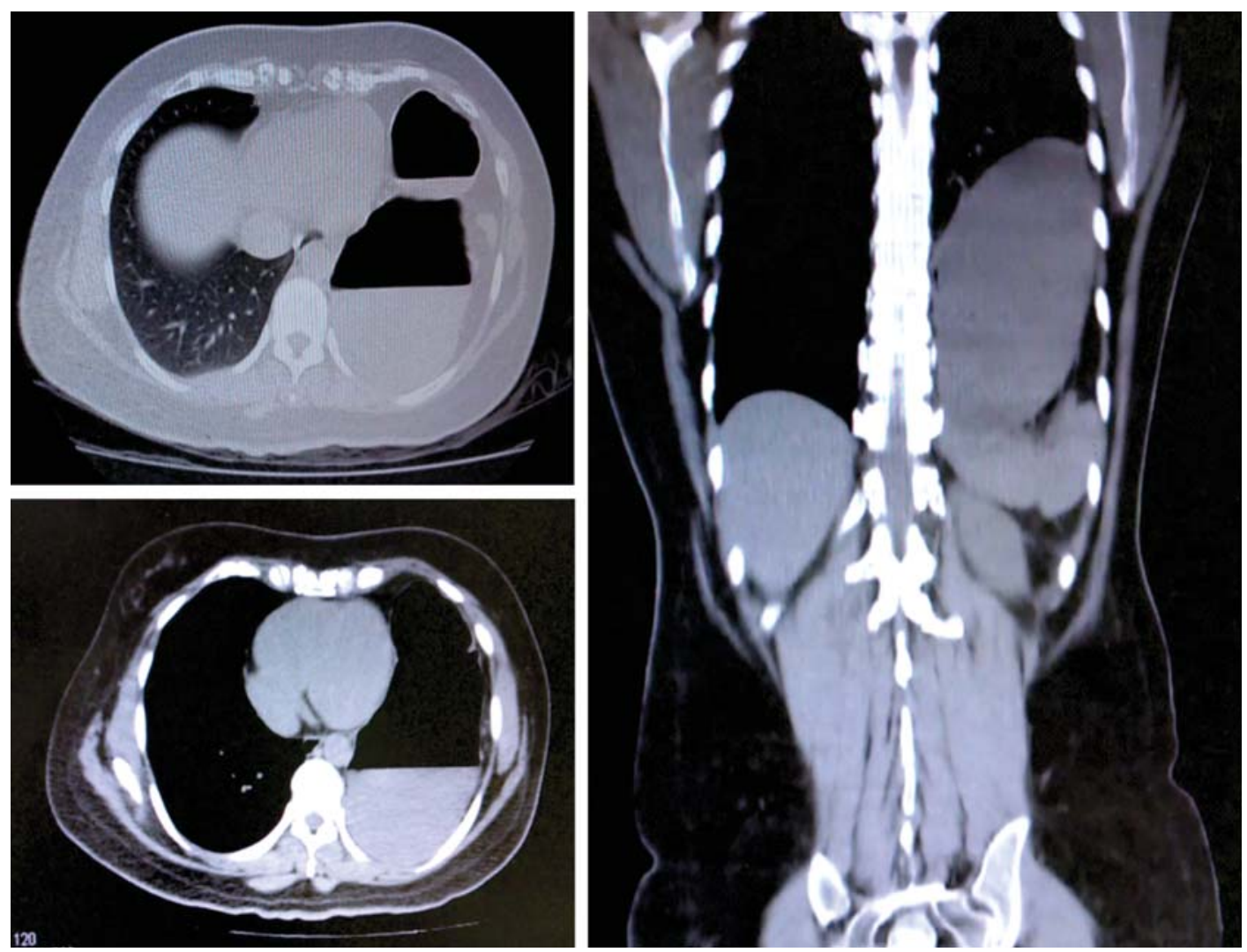
de poliéster trenzado calibre dos ceros, realizando puntos simples al diafragma. Se colocó una sonda intrapleural izquierda. La paciente permaneció los primeros tres días postoperatorios en la Unidad de Cuidados Intensivos; posteriormente, fue trasladada a hospitalización general. Egresó al décimo día postoperatorio sin eventualidades.

\section{DISCUSIÓN}

La mayoría de las hernias de Bochdalek son diagnosticadas durante la edad neonatal; se presentan con síntomas de dificultad respiratoria severa acompañada de hipoplasia pulmonar e hipertensión pulmonar.

La mortalidad a esta edad se encuentra entre 40 y $50 \%$. La mayoría de los individuos adultos se encuentran asintomáticos y su diagnóstico se realiza de manera incidental. ${ }^{7}$ Disnea crónica, dolor torácico y sensación de plenitud postprandial son los síntomas más comunes en los adultos. ${ }^{8}$

Existen diferentes modalidades para realizar el diagnóstico; entre ellas se encuentran la radiografía simple de tórax, la tomografía computada simple o contrastada, la resonancia magnética y ultrasonido toracoabdominal. ${ }^{8} \mathrm{El}$ diagnóstico inicial en la mayoría de las ocasiones se realiza con radiografía simple de tórax debido a la alta sensibilidad.

La tomografía contrastada se considera el estándar de oro para el diagnóstico en los casos electivos y de urgencias; en el estudio de Killean y sus colaboradores, se encontró sensibilidad en algunas series reportadas de hasta $78 \%$ para el lado izquierdo y $50 \%$ para el derecho; el signo del collar se presenta frecuentemente y tiene una sensibilidad del $36 \%{ }^{9}$

La reparación de la hernia puede realizarse vía transtorácica o abdominal, actualmente por mínima invasión o de forma convencional. ${ }^{9} \mathrm{La}$ vía transabdominal da la ventaja al cirujano de confirmar la posición de las vísceras abdominales y su compromiso; el procedimiento de elección dependerá del cirujano y su experiencia. Las técnicas mínimamente invasivas se han relacionado con disminución de la morbilidad, menor estancia hospitalaria, menor tiempo de recuperación y mayor beneficio cosmético. ${ }^{9}$ De igual manera, se ha debatido por mucho tiempo cuál es la mejor opción para el cierre del defecto: el cierre directo o el uso de material protésico; desde 1990, el uso de este último ha sido más común, sin embargo en la literatura el uso de malla protésica sólo ha sido utilizado en seis estudios de los 32 llevados a cabo, en todos los demás se realizó cierre directo del defecto; el uso de material protésico es recomendado por algunos autores cuando el defecto mide más de $10 \mathrm{~cm}$; no obstante, se ha reportado con mayor índice de complicaciones como infección, fístulas intestinales, migración de la malla y perforación entre muchas otras. ${ }^{7}$

\section{CONCLUSIONES}

La hernia de Bochdalek sintomática en una persona adulta representa, por la poca frecuencia de esta patología, un reto diagnóstico para el cirujano, y debe ser tomada en cuenta como uno de los diagnósticos diferenciales de dolor abdominal. El tratamiento siempre es quirúrgico y puede optarse actualmente por cirugía de mínima invasión o convencional, así como por un abordaje transtorácico o abdominal. La elección del tipo de abordaje para la reparación de la hernia dependerá de la experiencia de cada cirujano. La presencia de una perforación gástrica en el estómago herniado hacia tórax es poco común. El diagnóstico adecuado y el tratamiento quirúrgico oportuno influyen en la evolución postoperatoria.

La técnica de reparación abdominal mediante un abordaje transabdominal subcostal permite una adecuada visualización del contenido abdominal $y$, a la vez, del defecto en el diafragma. La técnica utilizada en nuestro caso consistió en el cierre de la perforación gástrica en dos capas y el cierre del defecto diafragmático mediante puntos simples con material de sutura no absorbible dos ceros. Este procedimiento es una técnica estandarizada con buenos resultados postoperatorios y un índice de complicaciones mínimo.

\section{REFERENCIAS}

1. Anil Luther AM. Left-side Bochdalek hernia in an adult: a case report with review of the literature. JMSA. 2015; 28: 33-34.

2. Herling A, Makhdom F, Al-Shehri A, Mulder DS. Bochdalek hernia in a symptomatic adult. Ann Thorac Surg. 2014; 98: 701-704. 
3. Hamid KS, Rai SS, Rodriguez JA. Symptomatic Bochdalek hernia in an adult. JSLS. 2010; 14: 279-281.

4. Faizah MZ, Sharifah MI, Johoruddin K, Juliana AL. Adult Bochdalek hernia complicated with perforated gastric ulcer: preoperative diagnosis with multiplanar CT. Med J Malaysia. 2011; 66: 367-368.

5. Sutedja B, Muliani Y. Laparoscopic repair of a Bochdalek hernia in an adult woman. Asian J Endosc Surg. 2015; 8: 354-356.

6. Rey DR. Hernia de Bochdalek. RAMR. 2015; 2: 137 138.

7. Brusciano L, Izzo G, Maffettone V, Rossetti G, Renzi A Napolitano $V$, et al. Laparoscopic treatment of Bochdalek hernia without the use of a mesh. Surg Endosc. 2003; 17: 1497-1498.
8. Singh TC, Singh CG, Lamare KN, Babitha N, Kharnaior A. Congenital diaphragmatic hernia in adult presenting with obstruction: a rare case. International Journal of Scientific Study. 2015; 2: 142-145.

9. Hung YH, Chien YH, Yan SL, Chen MF. Adult Bochdalek hernia with bowel incarceration. J Chin Med Assoc. 2008; 71: 528-531.

\section{Correspondencia:}

Dr. Jorge Favio Lezama de Luna

Av. Benito Juárez $\mathrm{S} / \mathrm{N}$,

San Nicolás de los Garza,

66424, Nuevo León, México.

Celular: 8110825580

E-mail: faviolezama@hotmail.com 